\title{
Environmental Interventions to Reduce the Consumption of Sugar-Sweetened Beverages: Abridged Cochrane Systematic Review
}

\author{
Peter von Philipsborn ${ }^{a, b}$ Jan M. Stratil ${ }^{a}$ b Jacob Burns ${ }^{a, b}$ \\ Laura K. Busert ${ }^{c}$ Lisa M. Pfadenhauer ${ }^{a}, b$ Stephanie Polus ${ }^{a}, b$ \\ Christina Holzapfel ${ }^{d}$ Hans Hauner ${ }^{d}$ Eva A. Rehfuess ${ }^{a}$ b \\ anstitute for Medical Information Processing, Biometry and Epidemiology, LMU Munich, \\ Munich, Germany; ${ }^{b}$ Pettenkofer School of Public Health, Munich, Germany; \\ 'Great Ormond Street Institute of Child Health, University College London, London, UK; \\ ${ }^{\mathrm{d}}$ Institute for Nutritional Medicine, Klinikum rechts der Isar, Technical University Munich, \\ Munich, Germany
}

\author{
Keywords \\ Sugar-sweetened beverages · Interventions - Systematic review · Cochrane
}

\begin{abstract}
Background: Regular consumption of sugar-sweetened beverages (SSB) can increase the risk for obesity, type 2 diabetes, cardiovascular disease, and dental caries. Interventions that alter the physical or social environment in which individuals make beverage choices have been proposed to reduce the consumption of SSB. Methods: We included randomised controlled, non-randomised controlled, and interrupted time series studies on environmental interventions, with or without behavioural co-interventions, implemented in real-world settings, lasting at least 12 weeks, and including at least 40 individuals. Studies on the taxation of SSB were not included, as these are subject of a separate Cochrane review. We used standard Cochrane methods for data extraction, risk of bias assessment, and evidence grading and synthesis. Searches were updated to January 24, 2018. Results: We identified 14,488 unique records and assessed 1,030 full texts for eligibility. We included 58 studies comprising a total of 1,180,096 participants and a median length of follow-up of 10 months. We found moderate-certainty evidence for consistent associations with decreases in SSB consumption or sales for the following interventions: traffic light labelling, price increases on SSB, in-store promotion of healthier beverages in supermarkets, government food benefit programs with incentives for
\end{abstract}


von Philipsborn et al.: Interventions to Reduce the Consumption of Sugar-Sweetened Beverages

purchasing fruits and vegetables and restrictions on SSB purchases, multi-component community campaigns focused on SSB, and interventions improving the availability of low-calorie beverages in the home environment. For the remaining interventions we found low- to verylow-certainty evidence for associations showing varying degrees of consistency. Conclusions: With observed benefits outweighing observed harms, we suggest that environmental interventions to reduce the consumption of SSB be considered as part of a wider set of measures to improve population-level nutrition. Implementation should be accompanied by evaluations using appropriate methods. Future studies should examine population-level effects of interventions suitable for large-scale implementation, and interventions and settings not yet studied thoroughly.

(C) 2020 The Author(s)

Published by S. Karger AG, Basel

\section{Introduction}

Sugar-sweetened beverages (SSB) - defined as non-alcoholic, non-dairy beverages with added caloric sweeteners - have been linked to a number of adverse health outcomes. There is evidence for a causal role of SSB in the development of overweight and obesity from randomised controlled trials (RCTs) in children and youth and from systematic reviews of randomised controlled and observational studies in various age groups [1-4]. Moreover, systematic reviews of observational studies have found positive associations between SSB intake and type 2 diabetes [5], cardiovascular disease [6], and dental caries [7].

SSB consumption varies considerably by geographic location, gender, age, and socioeconomic status. Based on a systematic review and pooled analysis of dietary surveys, global mean adult daily SSB consumption was estimated at $137 \mathrm{~mL}$ (95\% CI 88-211 mL) in 2010 [8]. Mean daily SSB consumption is higher in upper-middle-income countries (189 mL) and lower-middle-income countries $(140 \mathrm{~mL})$ than in high-income $(121 \mathrm{~mL})$ and low-income (83 $\mathrm{mL}$ ) countries, with substantial variation between countries [8]. Among the populations with the highest intake, SSB constitute a substantial source of energy. In the US, mean SSB consumption in 2010 was estimated to account for 8 and $7 \%$ of total energy intake among youth and adults respectively [9]. In most high-income countries, SSB intake is higher among disadvantaged than among relatively more privileged population groups [10].

Public health and medical organisations have therefore called for efforts to decrease population-level SSB consumption. This includes environmental interventions, which target the physical and social environments in which individuals make food and beverage choices, as well as behavioural interventions, which target individuals and seek to alter their dietary preferences, knowledge, attitudes, motivations, skills, and abilities [11]. Systematic reviews that included RCTs and observational studies on behavioural interventions [12-14], and systematic reviews of observational studies on SSB taxes have concluded that these can be effective in reducing SSB intake [15]. Less is known on other types of environmental interventions. In this summary of a Cochrane systematic review [16], we therefore examine the effects of non-tax environmental interventions to reduce the consumption of SSB.

\section{Methods}

This is an abridged version of a Cochrane systematic review. The protocol [17] and Cochrane review [16] are available open access from the Cochrane Library, and provide a detailed description of methods and results. Ethical approval was not required for this research. 
von Philipsborn et al.: Interventions to Reduce the Consumption of Sugar-Sweetened Beverages

Inclusion Criteria

Online supplementary File 1, Table S1 (for all online suppl. material, see www.karger. com/doi/10.1159/000508843) provides an overview on inclusion criteria.

Study Type

We included the following study types, as recommended by the Cochrane Effective Practice and Organization of Care (EPOC) Group [18]: RCTs, non-randomised controlled trials (NRCTs), controlled before-after (CBA) studies, interrupted time series (ITS) studies, and repeated measures studies (RMS). For the classification of study designs, we used the algorithm provided by EPOC [18]. We included only study designs that satisfy the following criteria:

- For cluster-RCTs, NRCTs, and CBA studies: studies with at least 2 intervention and 2 control sites.

- For ITS studies and RMS: a clearly defined point in time when the intervention occurred and at least 3 data points before and 3 data points after the intervention.

We included non-randomised study designs in order to cover a broader set of study populations, intervention types, and intervention contexts.

\section{Participants}

Any participants, including adults, adolescents, and children, regardless of their weight and health status. We included studies that collected data on individual participants, and studies based on data not linked to individual participants, such as sales data.

\section{Interventions}

We included environmental interventions, defined as interventions that aim to alter the food and beverage environment in a way which can, in principle, be permanent. Interventions that alter the food and beverage environment temporarily with the aim of influencing individual preferences (e.g., one-off public media campaigns) were considered as behavioural interventions and were not included in this review.

We excluded the following:

- Studies on the taxation of SSB, as these are the subject of a separate Cochrane review [19]

- Studies conducted in laboratory or virtual settings, as the results may not be generalisable to real-world environments

- Studies with a follow-up period of $<12$ weeks, as the effects may not be sustained

- Very small studies with $<20$ individuals in each the intervention or control group, as the results may not be generalisable

- Studies in which participants are administered pre-defined amounts of SSB or alternatives to SSB, as these studies provide only limited evidence on the feasibility and effectiveness of public health interventions aimed at reducing SSB intake among free-living individuals

- Studies on interventions not focused on SSB and not reporting beverage-specific outcome measures, as it would be difficult to attribute effects to the intervention components of interest

We defined SSB as non-alcoholic, non-dairy beverages with added caloric sweeteners. Based on feedback from members of our review advisory group (listed in online suppl. File 1, Table S2), we also included studies targeting sugar-sweetened milk, but analysed these separately. Dietary guidelines generally recommend to choose unsweetened milk [20], but it remains controversial whether sugar-sweetened milk should be targeted in public health interventions or not [21]. It has been argued that limits on the consumption of sugarsweetened milk could decrease overall milk intake, and thus overall dietary quality [21]. We 
von Philipsborn et al.: Interventions to Reduce the Consumption of Sugar-Sweetened Beverages

therefore considered interventions targeting sugar-sweetened milk separate from those targeting SSB and considered effects on total milk intake as potential adverse outcome [21].

$100 \%$ fruit juice (i.e., fruit juice without added sugars) can have a sugar content similar to that of many SSB and may have adverse health effects when consumed excessively [22]. It can, however, also be a relevant source of essential nutrients, in particular for populations with limited access to fresh fruits and vegetables [22]. Based on feedback from our Review Advisory Group, we therefore decided not to consider interventions targeting 100\% fruit juice in this review.

The comparison was no, minimal, or alternative intervention, such as behavioural intervention only.

Outcomes

We included studies reporting effects on direct or indirect measures of SSB and sugarsweetened milk intake (including consumption, purchasing, and sales data), diet-related anthropometric measures and health outcomes, or any adverse outcome or unintended consequence.

\section{Search Methods}

We searched 11 general, specialist, and regional databases, including: MEDLINE, Embase, CENTRAL, Scopus, Google Scholar, Social Science Citation Index, eLENA, openGrey, SciELO Citation Index, and LILACS. We used Scopus to search reference lists and citations of existing reviews and included studies. We scanned websites of relevant organisations and contacted study authors and members of our review advisory group. To identify ongoing or completed, but unpublished studies, we searched the WHO International Clinical Trials Registry Platform (ICTRP) and ClinicalTrials.gov. No date or language restrictions were applied, and electronic searches were updated to January 24, 2018 (by P.P.). The full search strategy is provided in online supplementary File 2.

\section{Study Selection}

Two review authors independently screened titles and abstracts of all studies, removing those which were clearly notrelevant. Subsequently, 2 review authors independently assessed full texts of all studies selected in step one for eligibility. Disagreement was resolved by discussion, and where necessary by consulting a third review author. We used EndNote and Zotero to collect and de-duplicate studies, the web application Rayyan for title and abstract screening, and Excel to document reasons for exclusion at full text screening stage. Screening was done by P.P., J.M.S., J.B., L.K.B., L.M.P., and S.P.

\section{Data Extraction and Management}

Two review authors (P.P., J.M.S., J.B., L.K.B., L.M.P., S.P., or C.H.) independently extracted study characteristics and study data with an electronic data extraction form (shown in online suppl. File 2). Inconsistencies were resolved by discussion, and where necessary by consulting a third review author. The final agreed data were entered into Review Manager 5.3 by P.P., and checked by J.M.S. We used the difference in means as main measure of effects. We extracted both adjusted and unadjusted results but used adjusted results for our evidence synthesis. In studies reporting several alternative measures for the same outcome, we used the outcome measure pre-specified as primary by the study in question, or alternatively the most conservative effect estimate reported. For studies reporting data on several time points, we used data for the last follow-up assessment conducted during the intervention phase. We extracted both data on the total number of participants recruited, enrolled or allocated, and on the number of participants included in analyses, and used the latter for our evidence synthesis. 
In cases in which missing data on study characteristics or outcome measures precluded study inclusion or limited the use of a study at further stages of the review, we contacted the corresponding author. For registered but unpublished trials, we contacted the corresponding investigator to request relevant data.

\section{Assessment of Risk of Bias}

Risk of bias of included studies was independently assessed by 2 review authors (P.P., J.M.S., J.B., L.K.B., L.M.P., S.P., or C.H.) with the Cochrane risk of bias tool adapted by EPOC for non-randomised study designs [18]. Inconsistencies were resolved by discussion, and where necessary by consulting a third review author.

\section{Data Synthesis}

We classified interventions with the NOURISHING framework into 7 broad intervention areas [11] and created in an ad hoc manner an eighth intervention area for home-based interventions. Based on feedback from our review advisory group, we further subdivided these 8 intervention areas in an ad hoc manner into 24 intervention types in order to improve the review's usefulness for decision-makers. The resulting typology of interventions is shown in online supplementary File 1, Table S3. We examined results for each of the 24 intervention types separately. Results were synthesised narratively and with tables. We conducted metaanalyses where methods and populations, interventions, comparisons, and outcomes were sufficiently homogenous.

\section{Assessment of the Certainty of Evidence}

We assessed our confidence in the certainty of evidence with the GRADE framework as very low, low, moderate, or high, using GRADE guidance [23]. In GRADE, there are 5 factors that can lead to a downgrading of the level of evidence (risk of bias, indirectness, inconsistency, imprecision, and publication bias) and 3 factors that can increase the level of evidence (magnitude of effect size, direction of plausible confounding, and dose-response gradient) [23]. In GRADE, the term "certainty of evidence" refers to our confidence that effects lie within a particular range, or on one side of a particular threshold [23]. For population-level public health interventions, any difference from the null may be potentially relevant, in particular for low-cost or cost-neutral interventions. We therefore used the difference from the null as threshold for the potential public health relevance of reported effects. The certainty of evidence therefore refers to our confidence that effects are different from the null. We did, however, document any cases in which reported effect sizes were small, and may arguably be of limited practical relevance. This was the case for 2 studies [24, 25].

\section{Public Involvement}

We formed a review advisory group with evidence users, including representatives of government agencies, international organisations, civil society, and academia, which were consulted at various stages of the review process. We have developed a dissemination plan involving our review advisory group and a variety of further stakeholders.

\section{Results}

Results of the Search

We identified 14,488 unique records and assessed 1,030 full texts for eligibility. Fiftyeight studies met our inclusion criteria, including 22 RCTs, 3 NRCTs, 14 CBA studies, and 19 ITS studies. Details on the search and screening process are shown in the PRISMA flow chart 
von Philipsborn et al.: Interventions to Reduce the Consumption of Sugar-Sweetened Beverages

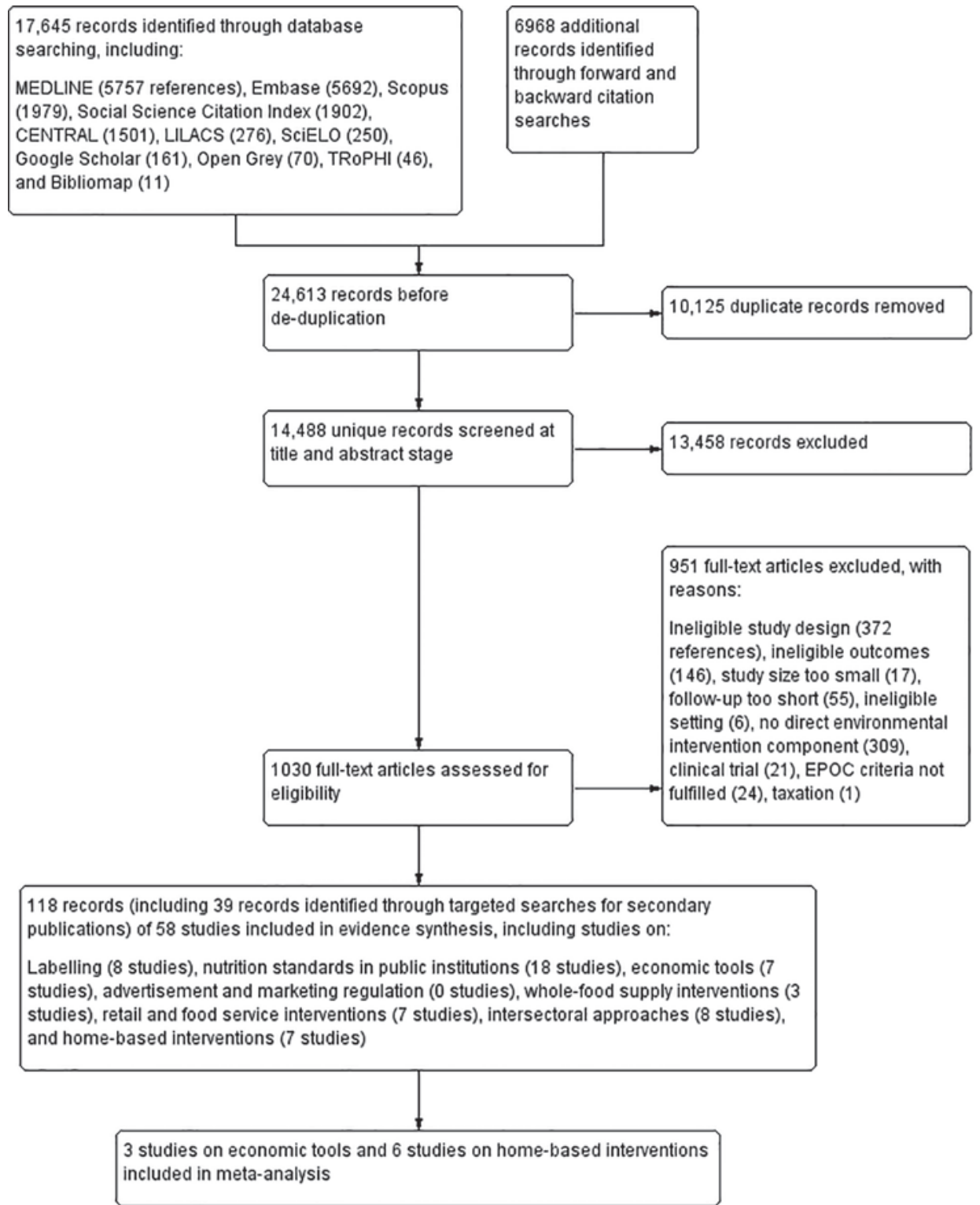

Fig. 1. PRISMA flow chart.

in Figure 1. Thirty-six studies collected data on individual participants, and 22 studies were based on sales data not linked to individual participants. The studies reporting participant numbers included a total of 1,180,096 participants (range 93-1,065,562, median 1,032). The combined length of intervention and follow-up ranged from 3 months to 6 years, with a median length of 10 months. Most studies were conducted in North America ( $n=40$ studies, $69 \%$ of included studies), Western Europe $(n=8,14 \%)$, and high-income Australasia $(n=6$, 10\%). Tables 1 and 2 provide an overview of methodological aspects and on participants, context, and implementation of included studies, respectively. 
Table 1. Characteristics of included studies - methods

\begin{tabular}{ll}
\hline Study design & $\begin{array}{l}\text { Studies, } n \text { (\% of included } \\
\text { studies) }\end{array}$ \\
\hline RCTs & $22(38)$ \\
ITS studies & $19(33)$ \\
CBA/NRCTs & $17(29)$ \\
Data collection methods (multiple counts) & $24(41)$ \\
$\quad$ Self-reported SSB intake & $22(38)$ \\
Electronic sales data & $8(14)$ \\
Production and sales data provided by market research firms & $3(5)$ \\
Observations by trained observers & $2(3)$ \\
Grocery and restaurant receipts collected from participants & $1(2)$ \\
$\quad$ Administrative data bases on beverage deliveries to schools & $36(62)$ \\
Type of outcome data & $22(38)$ \\
$\quad$ Data linked to individual participants & \\
Transaction data, not linked to individual participants &
\end{tabular}

RCT, randomised controlled trial; ITS, interrupted time series study; CBA, controlled before-after study; NRCT, non-randomised controlled trial.

Table 2. Characteristics of included studies - participants, context, and implementation

\begin{tabular}{lc}
\hline Age group of participants & $\begin{array}{l}\text { Studies, } n \text { (\% of included } \\
\text { studies) }\end{array}$ \\
\hline Children & $12(21)$ \\
Children and teenagers & $8(14)$ \\
Teenagers & $7(12)$ \\
Adults & $9(16)$ \\
Not specified/participants of all ages & $22(38)$ \\
Setting & \\
Schools & $20(34)$ \\
Retailing & $12(21)$ \\
Homes & $7(12)$ \\
Food service & $6(10)$ \\
Other & $13(22)$ \\
Country & \\
US & $38(66)$ \\
Australia & $4(7)$ \\
The Netherlands & $3(5)$ \\
Canada & $2(3)$ \\
UK & $2(3)$ \\
Other & $9(16)$ \\
Mode of implementation & \\
Pilot trial by researchers & $29(50)$ \\
Mandatory government regulation & $16(28)$ \\
Voluntary industry action & $9(16)$ \\
Government pilot project & $4(7)$ \\
&
\end{tabular}

\section{Risk of Bias and Certainty of Evidence}

We judged most studies to be of high or unclear risk of bias in at least one domain (see online suppl. File 1, Fig. S1, 2). For a number of intervention types and outcomes most or all included studies reported effect estimates with 95\% CIs that were large and included both 

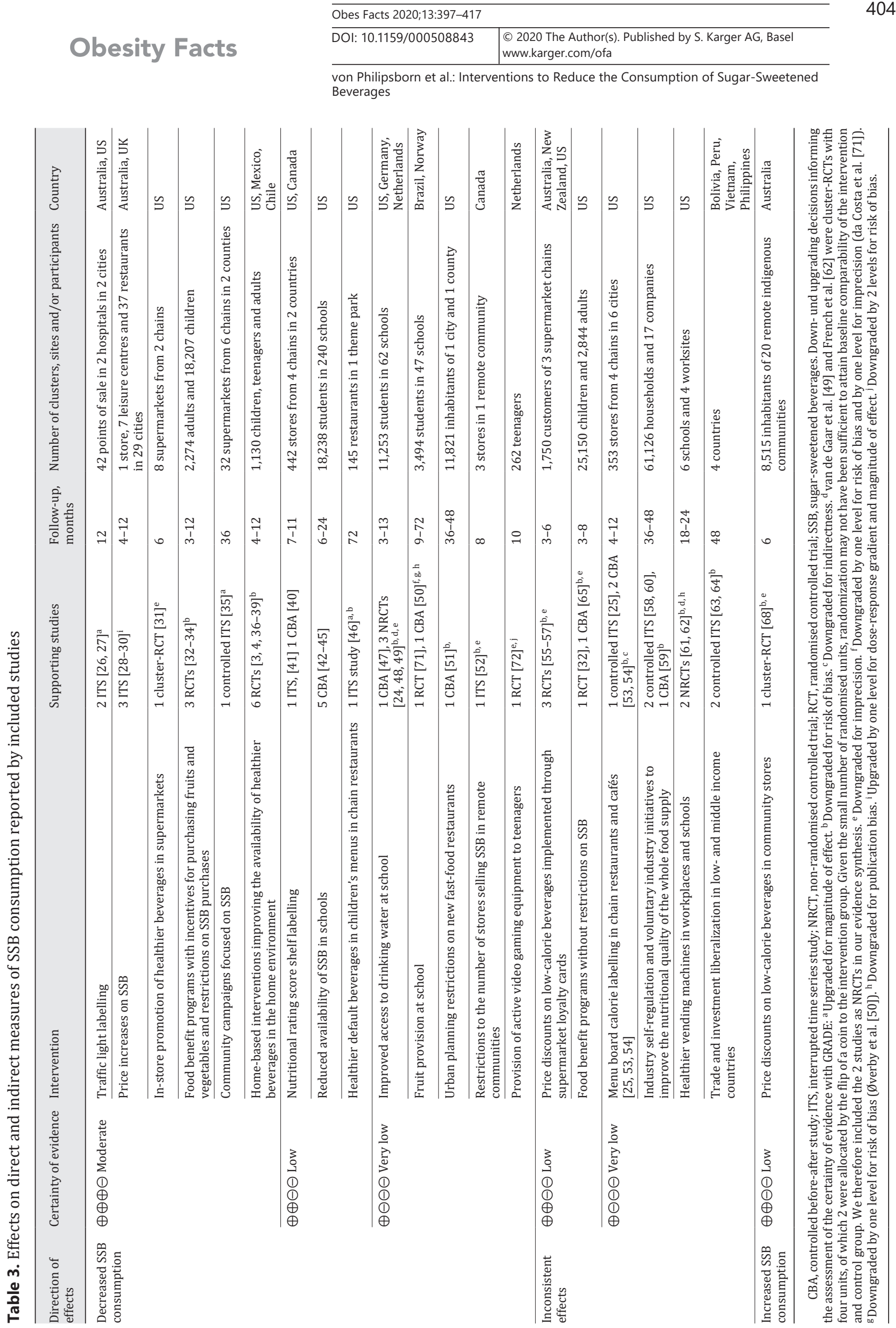
von Philipsborn et al.: Interventions to Reduce the Consumption of Sugar-Sweetened Beverages

Table 4. Effects on body weight outcomes reported by included studies

\begin{tabular}{|c|c|c|c|c|c|c|}
\hline $\begin{array}{l}\text { Direction } \\
\text { of effects }\end{array}$ & $\begin{array}{l}\text { Certainty of } \\
\text { evidence }\end{array}$ & Intervention & Supporting studies & $\begin{array}{l}\text { Follow-up, } \\
\text { months }\end{array}$ & $\begin{array}{l}\text { Number of clusters, } \\
\text { sites and/or participants }\end{array}$ & Country \\
\hline \multirow[t]{3}{*}{$\begin{array}{l}\text { Decrease in } \\
\text { body weight }\end{array}$} & $\oplus \oplus \oplus \oplus$ High & $\begin{array}{l}\text { Improved access to low-calorie } \\
\text { beverages in the home environment, } \\
\text { compared to no intervention }\end{array}$ & 2 RCTs $[3,4]$ & $6-12$ & 320 teenagers & US \\
\hline & $\oplus \oplus \ominus \ominus$ Low & $\begin{array}{l}\text { Improved access to low-calorie } \\
\text { beverages in the home environment } \\
\text { compared to general weight loss } \\
\text { counselling }\end{array}$ & 2 RCTs $[38,39]^{a, b}$ & $6-9$ & 558 adults & US \\
\hline & $\oplus \ominus \ominus \ominus$ Very low & $\begin{array}{l}\text { Improved access to drinking water } \\
\text { in schools }\end{array}$ & $\begin{array}{l}1 \text { NRCT [48], } 1 \text { CBA [66], } \\
\text { and } 1 \text { cluster RCT }[49]^{\mathrm{a}, \mathrm{b}}\end{array}$ & $10-60$ & $\begin{array}{l}1,069,521 \text { students at } \\
1,231 \text { schools }\end{array}$ & $\begin{array}{l}\text { US, Germany, } \\
\text { Netherlands }\end{array}$ \\
\hline
\end{tabular}

CBA, controlled before-after study; NRCT, non-randomised controlled trial; RCT, randomised controlled trial. Down- und upgrading decisions informing the assessment of the certainty of evidence with GRADE: ${ }^{a}$ Downgraded for risk of bias. ${ }^{b}$ Downgraded for imprecision.

the possibility of relevant benefit and null effects or harm. We therefore downgraded the level of evidence for most interventions and most outcomes for risk of bias, imprecision, or both [23]. For 3 intervention types (school fruit programs, healthier vending machines, and provision of active video gaming equipment), we downgraded the certainty of evidence for suspected publication bias, as we identified studies on these intervention types that had assessed but did not fully report effects on SSB intake. In one case (menu board calorie labelling), we downgraded the certainty of evidence for indirectness, as all studies providing data on this intervention type reported only an indirect measure for SSB intake, namely calories from beverages per transaction. In 4 cases (traffic light labelling, price increases on SSB, healthier default beverages in children's menus in chain restaurants, and community campaigns focused on SSB), we judged the observed effect size to be large, and unlikely to have arisen by chance or due to bias, and upgraded the certainty of evidence for this reason. Our confidence in the certainty of most effect estimates showing a non-null effect is therefore moderate, low, or very low. Details on the assessment of the certainty of evidence for each outcome and each intervention type are provided in the footnotes to Tables 3-5 and in the full Cochrane review [16].

\section{Effects on SSB Consumption}

Heterogeneity in methods as well as in population, intervention, comparison, and outcomes precluded meta-analysis for most outcomes and intervention types. The direction of reported effects on SSB consumption and body weight, as well as key aspects of the supporting evidence are shown in Tables 3 and 4, respectively. Full quantitative results for all outcomes and all intervention types are shown in the Cochrane version of this review.

For 6 intervention types, we found moderate-certainty evidence for consistent associations with decreases in direct or indirect measures of SSB consumption: traffic light labelling $[26,27]$; price increases on SSB [28-30]; in-store promotion of healthier beverages in supermarkets [31]; food benefit programs with incentives for purchasing fruits and vegetables and restrictions on SSB purchases [32-34]; community campaigns focused on SSB [35]; and interventions improving the availability of healthier beverages in the home environment $[3,4$, 36-39].

We found low-certainty evidence for consistent associations with decreases in SSB consumption for: nutritional rating score shelf labelling [40,41]; limits on the availability of SSB in schools [42-45]; and healthier default beverages in children's menus in chain restaurants [46]. 
von Philipsborn et al.: Interventions to Reduce the Consumption of Sugar-Sweetened Beverages

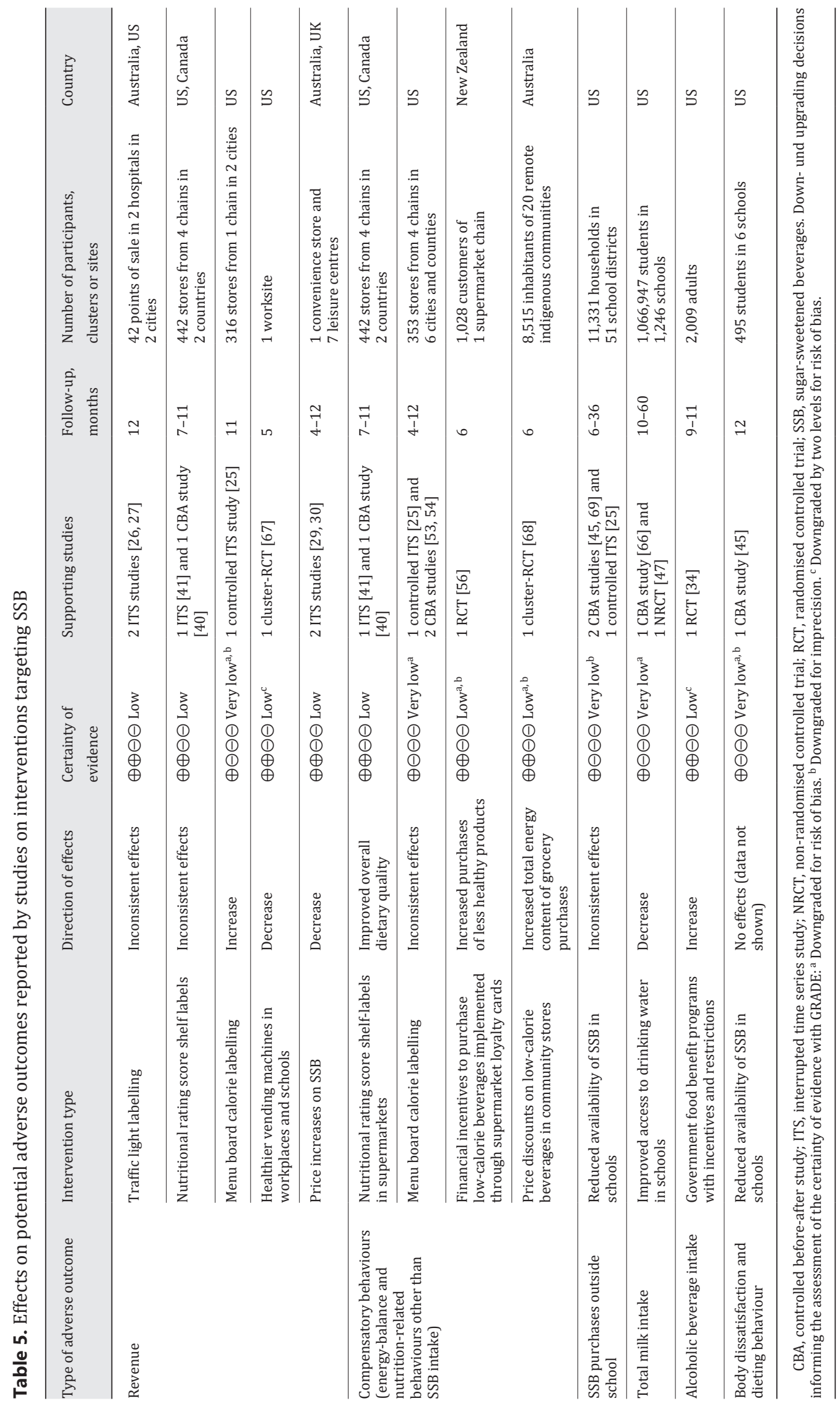


We found very low-certainty evidence for associations with decreases in SSB consumption for: improved access to drinking water at school [24, 47-49]; fruit provision at school [50]; urban planning restrictions on new fast-food restaurants [51]; and restrictions on the number of stores selling SSB in remote communities [52].

For the following intervention types, we found inconsistent associations with SSB consumption: menu board calorie labelling in chain restaurants and cafés [25, 53, 54]; price discounts on low-calorie beverages implemented through supermarket loyalty cards [5557]; industry self-regulation and voluntary industry initiatives to improve the nutritional quality of the whole food supply [58-60]; healthier vending machines in workplaces and schools $[61,62]$; trade and investment liberalisation in low- and middle-income countries $[63,64]$; and food benefit programs without restrictions on SSB [32, 65].

Moreover, we found high-certainty evidence that interventions improving the availability of low-calorie beverages in the home environment of adolescents with a high baseline consumption of SSB are associated with a smaller gain in body weight compared to no intervention $[3,4]$. We found low-certainty evidence that such interventions are associated with reduced body weight among adults when compared with general weight loss counselling [38, 39]. Moreover, we found very low-certainty evidence that improved access to drinking water at school may be associated with reduced body weight $[48,49,66]$.

We conducted a meta-analysis for 3 studies reporting on price increases of SSB [28-30]. Our pooled effect estimate is that SSB sales decreased by $-19 \%(95 \%$ CI -33 to -6$)$ at $4-12$ months, with relevant subgroup differences according to the magnitude of the price increase (see online suppl. File 1, Fig. S3). Moreover, we conducted meta-analyses for 5 studies reporting effects on SSB consumption of improved access to low-calorie beverages in the home environment of individuals with a high baseline consumption of SSB [3, 4, 36-38]. Across these studies, SSB consumption decreased by $-413 \mathrm{~mL} /$ day (95\% CI -684 to -143$)$ at 4-12 months (see online suppl. File 1, Fig. S4). Here, however, the graphical inspection of the forest plot and the $I^{2}$ statistic indicate substantial statistical heterogeneity, which may be explained by differences in population, intervention delivery, and context.

\section{Adverse Outcomes and Unintended Consequences}

Table 5 presents evidence on potential adverse outcomes and unintended consequences reported by included studies. These include effects on revenue [25-27, 29, 30, 40, 41, 67], various types of compensatory behaviour (defined as energy balance and nutrition-related behaviours other than SSB consumption, which may be directly or indirectly affected by the intervention) $[25,40,41,53,54,56,68]$, as well as SSB purchases outside school when availability of SSB in schools is reduced [25, 45,69]. We included effect estimates for these outcomes regardless of the reported direction of effect. Reported effects varied considerably. For example, 5 studies on labelling examined effects on total revenue, and one study each reported statistically significant positive [41] and negative [26] effects, while 3 studies reported effects not reaching statistical significance at the $5 \%$ level $(2$ positive $[25,27]$, and one negative effects [40]). Similarly, 3 studies examined effects on SSB purchases and consumption outside school when access to SSB at school is limited, and one study found a statistically significant increase for high-school students but decreases for middle and elementary school children [70], while 2 other studies examining middle schools [45] and a variety of different school types [69], respectively, found decreases that did not reach statistical significance at the $5 \%$ level.

\section{Effects by Indicators of Social Disadvantage}

Among the studies included in this review, 13 focused on socially disadvantaged participants [31-33, 48, 49, 51, 57, 65, 68, 73-75], and 9 report subgroup analyses by indicators of social disadvantage $[3,4,25,48-50,53,55,56]$. We present details on these in the full 
von Philipsborn et al.: Interventions to Reduce the Consumption of Sugar-Sweetened Beverages

Cochrane review [16]. In short, we found no clear pattern of differences in effectiveness by social disadvantage, but some indication that interventions can be effective in groups exposed to varying degrees and forms of social disadvantage.

\section{Effects on Sugar-Sweetened Milk Consumption}

We analysed results for interventions targeting sugar-sweetened milk separately. We found low- [74, 76] to moderate-certainty [77] evidence that small prizes for the selection of healthier beverages in elementary school cafeterias are associated with decreased consumption of sugar-sweetened milk, and low-certainty evidence that this is the case for emoticon labelling [73]. We found very-low-certainty evidence that improved access to drinking water in schools is associated with decreased intake of sugar-sweetened milk [66] and that improved placement of plain milk in school cafeterias is not associated with sugarsweetened milk consumption [75]. Three studies on sugar-sweetened milk examined total milk intake $[73,74,76]$, and reported effects varied in direction, size, and statistical significance.

\section{Ongoing Studies}

We conducted trial register searches to identify ongoing studies and found 10 studies which we judged likely to meet our eligibility criteria upon completion. This includes 4 studies on improved availability of drinking water in schools [78-81], 2 studies on improved availability of low-calorie beverages in the home environment $[82,83]$, and one study each on reduced availability of SSB and improved availability of drinking water in schools [84], reduced availability of SSB in workplaces [85], price increases on SSB [86], and the removal of SSB from supermarket checkout coolers [87]. Trial register searches may, however, provide only an incomplete picture of ongoing research in the field, given that only a small share of all completed studies included in this review reported registration. We contacted authors of all ongoing studies, but preliminary outcome data was not available for any of them. We therefore did not include these in our analysis.

\section{Discussion}

\section{Principal Findings}

The evidence included in this review shows that effective, scalable interventions addressing SSB consumption on a population level exist. With the observed benefits outweighing the observed harms, we suggest that those interventions showing consistent associations with reduced SSB consumption may be considered as part of a wider set of measures to improve population-level nutrition and associated health outcomes. Based on the findings of this review, this applies to the following approaches: interpretative nutrition labelling, including traffic light [26, 27] and nutritional rating score labelling [40, 41]; interventions limiting the availability of SSB in schools [42-45]; price increases on SSB [28-30, 42-45]; healthier default beverages in children's menus in chain restaurants [46]; in-store promotion of healthier beverages in supermarkets [31]; government food benefit programs with incentives to purchase fruits and vegetables and restrictions on purchasing SSB [32-34]; multi-component community campaigns focused on SSB [35]; and interventions improving access to low-calorie beverages in the home environment of participants with overweight and obesity and a high baseline consumption of SSB [3, 4, 36-39]. Further approaches to reduce the consumption of SSB exist, and may be effective, but the evidence in this review does not allow for clear conclusions regarding their effects. Behavioural interventions and taxation of SSB were not examined in this review.

\section{Karger's}


von Philipsborn et al.: Interventions to Reduce the Consumption of Sugar-Sweetened Beverages

Certainty of Evidence, Applicability, and Interpretation of Results

Based on our GRADE ratings, the certainty of evidence is low to moderate for most interventions and in relation to most outcomes. This means that future studies may find effects which may be substantially different in size and possibly direction from those reported in this review. When examining the existing evidence, decision makers should, however, consider that in public health, the effects of population-level interventions can rarely be established conclusively by conducting trials in controlled research settings [88]. Generating meaningful evidence on such interventions generally requires that policy makers and practitioners take action based on limited and imperfect evidence and implement interventions while ensuring that these are evaluated with appropriate methods, and reviewed, modified, or discontinued once stronger evidence on population-level effects emerges [88].

The studies included in this review cover a broad range of settings, including schools, supermarkets, restaurants and hospitals, among others. Twenty-three of the included studies were implemented on the level of political jurisdictions such as states or municipalities. We included only studies conducted in real world settings (excluding studies conducted in research laboratories and virtual environments) lasting at least 3 months. This strengthens our confidence that, overall, results are applicable to a variety of real world settings, and are scalable to a population level. For a number of intervention types, however, the evidence in this review is limited to specific settings, modes of delivery, or populations. Both studies on traffic light labelling, for example, were implemented in hospitals and included a number of co-interventions, such as awareness-raising campaigns $[26,27]$. Only 4 studies were conducted in lowand middle-income countries [38,63,64,71], and 38 of the 58 studies included in the review were conducted in the USA. The heterogeneity in effects observed for most interventions and outcomes may be partly due to differences in methods, population, intervention, comparison, and outcome measures, but may also be a sign of the context dependency typical of public health interventions. Implementation of interventions in contexts different from those in which they have been examined so far may therefore require adaptation and may produce effects different from those reported in this review. This also applies to those interventions for which this review found inconsistent effects on SSB intake, including, for example, menu board calorie labelling $[25,53,54]$ and voluntary industry initiatives [58-60]. These may still constitute worthwhile approaches when adapted to a given context, and future studies may find them to be effective. Importantly, the evidence in this review does not allow to rule out relevant effects for any of the included intervention types. The absence of evidence for effects of some interventions should not be interpreted as evidence for the absence of effects.

\section{Comparison with Other Studies}

In a targeted search for existing systematic reviews on SSB interventions conducted on May 25 , 2018, we identified 5 systematic reviews that included environmental interventions to reduce the consumption of SSB other than taxation, including 4 that are focused on children and adolescents [14, 89-91], and one which includes interventions targeting all age groups [13]. There is substantial variation in the focus, the search strategies, the eligibility criteria and various other methodological aspects used by the 5 existing systematic reviews and our review. Accordingly, the overlap among the studies included in these reviews is small: only 11 of the 58 studies included in our review are also included in one of the existing systematic reviews. Overall, the results of the 5 existing systematic reviews indicate that environmental interventions can contribute to reductions in SSB intake and are thus in line with the findings of our review.

\section{Implications for Research and Practice}

A broad range of factors interact to influence SSB consumption, including accessibility, availability, and price of SSB as well as population attitude, awareness, and social norms

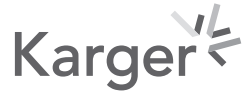


von Philipsborn et al.: Interventions to Reduce the Consumption of Sugar-Sweetened Beverages

related to the consumption of SSB and other sugary products, and SSB are consumed on a variety of occasions and locations [92]. It is therefore unlikely that any single intervention taken alone can effectively lower population-level SSB consumption to recommended levels [88]. It is encouraging that, as shown in this review, a broad range of interventions, implemented on a variety of levels by a variety of actors, can affect SSB consumption. Combining different approaches, and targeting a variety of contextual factors, settings, and population groups is likely to be necessary for bringing about the intended more substantial behavioural and health impacts [88].

Most of the interventions examined in this review are suitable for large-scale implementation and are likely to show the greatest population-level effect when implemented on this level. This, however, requires public and political support. The potential effectiveness of interventions in relation to their intended outcomes is only one of many factors influencing the likelihood of implementation. Adverse outcomes, in particular, feature prominently in the public and political debates around SSB interventions. It is therefore of relevance that we did not find consistent evidence for negative effects on outcomes such as stakeholder revenue or compensatory behaviours. Moreover, a sense of political dynamics, effective advocacy, and broad coalitions may help to achieve policy-level implementation, as described by Schwartz et al. [35], a study on a community campaign focused on SSB. While policy-level implementation may be desirable in most cases, implementation within specific settings, including hospitals [26, 27], may in many cases be a worthwhile and politically more viable option in the short-run. Moreover, when setting-level implementation is accompanied by evaluations using appropriate methods, it can support policy-level implementation by generating evidence, public visibility, and political momentum.

Conducting methodologically rigorous evaluations of population-level public health interventions poses unique challenges, and some of the studies in this review provide examples of how these can be addressed. Given that the implementation of such interventions often falls outside the capacity and authority of researchers, close and continuous cooperation with relevant stakeholders, such as public officials $[30,47,66]$, civil society organisations [35], and private businesses [25, 28, 29, 31, 40, 41] is often required. Randomisation may be feasible in some cases [68]. Where this is not the case, relatively more sophisticated non-randomised study designs, such as interrupted time series studies $[25,26,28-30,35,52$, 70] and designs with synthetic control groups [44] may be used. Data limitations may be addressed by making optimal use of existing data sources, including routinely collected electronic sales data $[25,26,28-31,40,41,52]$ and sales and purchasing data provided by commercial market research firms [35, 58-60, 63, 64, 69, 70]. Effects of public health interventions may vary by socio-economic status, gender, ethnicity, and other dimensions of social (dis-)advantage, and future studies should pay tribute to such differential effects. Pre-registration is still uncommon in the field - none of the 38 non-randomised studies in our review reported registration - but is desirable for all study types. Most study registers, including clinicaltrials.gov, allow for the registration of observational studies, including studies conducted retrospectively with routinely collected data. When pre-registration is not considered feasible, publication of protocols $[4,33,68]$ and declarations on protocol fidelity $[28,33]$ may help to alleviate concerns regarding reporting bias.

\section{Limitations of This Review}

This review has a number of limitations. We did not include studies on taxation of SSB and on behavioural (educational) interventions, as these are covered by existing or ongoing reviews $[12-15,19]$. Moreover, we did not find any studies meeting our inclusion criteria on a number of approaches proposed for reducing the consumption of SSB or its effects on health, including:

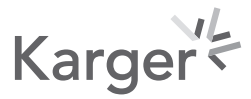


von Philipsborn et al.: Interventions to Reduce the Consumption of Sugar-Sweetened Beverages

- Advertisement and marketing regulations [93]

- Portion and package size reductions [94]

- Warning labels [95]

- National strategies to improve the quality of the whole food supply [96]

- Subsidies for and regulation of the production of sugar and other caloric sweeteners [97]

- Improved access to healthier beverages in small neighbourhood shops [98]

While studies have been conducted on these approaches, these did not meet our inclusion criteria with regard to follow-up [94, 95], outcomes [93, 96], or study design [97, 98].

Moreover, we conducted searches in English only, and note that 47 of the 58 included studies were conducted in Anglophone countries. We may have missed eligible studies published in languages other than English. We made a number of changes to the review's methodology after we had published the protocol and provide details on and the rationale for these changes in the full review published in the Cochrane Library [16]. We used reporting on primary outcomes as one eligibility criterion for studies, which limits our ability to ascertain the extent of reporting bias. We included studies reporting indirect measures of SSB consumption, including measures that are not specific to SSB, such as beverage calories per transaction [25, 53, 54] and energy density of beverages sold [56]. For a number of intervention types, the number of supporting studies was small and limited to specific settings and modes of implementation. We included studies on environmental interventions with behavioural co-interventions, which are likely to have influenced the observed effects. The classification of interventions as environmental or behavioural was in some cases ambiguous and required judgement by the review authors. We provide further details on these cases in the full review published in the Cochrane Library [16]. Our choice of 12 weeks as minimum length of follow-up required for inclusion may be considered arbitrary, and still represents a relatively short-term follow-up period. We used the Cochrane risk of bias tool adapted for non-randomised studies to assess risk of bias [18]. Using a more sophisticated tool, such as ROBINS-I, may have allowed us to better account for the relative strengths and weaknesses of different non-randomised study designs, both in the risk of bias assessment of individual studies and in the assessment of the certainty of evidence with GRADE.

\section{Conclusions}

Environmental interventions can help reduce population-level SSB consumption and should be considered as part of wider strategies to improve population-level nutrition. Their implementation should be accompanied by evaluations using appropriate study methods to strengthen the existing evidence base. There is a particular need for studies on populationlevel effects of interventions suitable for large-scale implementation, and on interventions, settings, and countries not yet examined thoroughly by existing studies.

\section{Acknowledgement}

We would like to acknowledge the substantial and generous contributions made by the members of our Review Advisory Group, who helped to inform the Background section and the parameters of the review at protocol stage. We would also like to acknowledge the helpful contributions by the Cochrane Public Health editorial team. We are grateful to Qëndresë Daka and Samuel Muli for their assistance with title and abstract screening.

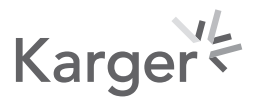




\section{Statement of Ethics}

Ethical approval was not required for this research.

\section{Conflict of Interest Statement}

All authors have completed the ICMJE uniform disclosure form at www.icmje.org/coi disclosure.pdf and declare: J.M.S., J.B., L.K.B., L.M.P., S.P., and E.A.R. have received support from LMU Munich for the submitted work; J.M.S. has received support from the Bavarian Office for Health and Food Safety for the submitted work; P.P., C.H., and H.H. have received support from Technical University Munich for the submitted work; C.H. is a member of the scientific advisory board of 4 sigma $\mathrm{GmbH}$, a consultancy working primarily for health insurance companies; C.H. has been involved in the preparation of a systematic review on Associations between single nucleotide polymorphisms and macronutrient intake (PROSPERO: CRD42015025738), partly funded by an institutional grant from the consumer products company Amway GmbH; H.H. has received honoraria for membership on scientific advisory boards of Weight Watchers, NovoNordisk and Boehringer Ingelheim, and honoraria for membership on scientific advisory boards of Nestle and Danone, providing advice on recommendations regarding the nutritional content of breakfast meals and dairy products; H.H.'s institution has received grants from Riemser Pharma, Amway GmbH and Certmedica, a producer of slimming products; no other relationships or activities that could appear to have influenced the submitted work.

\section{Funding Sources}

No external funding was received for this work. Internal funding was provided by LMU University of Munich, Germany (P.P., J.M.S., J.B., L.K.B., L.M.P., S.P., and E.A.R.), Technical University Munich, Germany (P.P., C.H., and H.H.), University College London, UK (L.K.B.) and the Bavarian Office for Health and Food Safety, Germany (J.M.S.). The research was conducted independently from the funder. P.P., J.M.S., J.B., L.K.B., L.M.P., S.P., and E.R. are employed by LMU University of Munich, J.M.S. is employed by the Bavarian Office for Health and Food Safety, and C.H. and H.H. are employed by the Technical University Munich.

\section{Author Contributions}

P.P. drafted the protocol with support from J.M.S., J.B., L.K.B., L.M.P., S.P., C.H., H.H., and E.A.R. P.P. developed the search strategy and conducted the searches. P.P., J.M.S., J.B., L.K.B., L.M.P., and S.P. selected studies, extracted data, and assessed the risk of bias of included studies. P.P. entered data into RevMan, conducted the analyses, graded the evidence, and drafted the manuscript. J.M.S. double-checked data entry, data analysis, and evidence grading. E.A.R. provided methodological support on all stages of the review process. P.P. is the guarantor of this manuscript. All authors had full access to all of the data (including statistical reports and tables) in the study and can take responsibility for the integrity of the data and the accuracy of the data analysis. The corresponding author attests that all listed authors meet authorship criteria and that no others meeting the criteria have been omitted.

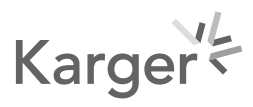




\begin{tabular}{l|l|}
\hline Obes Facts 2020;13:397-417 \\
\hline DOI: 10.1159/000508843 & $\begin{array}{l}\text { ○ } 2020 \text { The Author(s). Published by S. Karger AG, Basel } \\
\text { www.karger.com/ofa }\end{array}$ \\
\hline
\end{tabular}

von Philipsborn et al.: Interventions to Reduce the Consumption of Sugar-Sweetened Beverages

\section{Data Sharing}

Full datasets can be obtained from the corresponding author at pphilipsborn@ibe.med. uni-muenchen.de.

\section{Transparency}

The lead author affirms that the manuscript is an honest, accurate, and transparent account of the study being reported; that no important aspects of the study have been omitted; and that any discrepancies from the study as planned and registered have been explained. A detailed description of all differences between the review and the published protocol is provided in the section "Differences between protocol and review" of the review's full version published in the Cochrane Database of Systematic Reviews.

\section{References}

1 de Ruyter JC, Olthof MR, Seidell JC, Katan MB. A trial of sugar-free or sugar-sweetened beverages and body weight in children. N Engl J Med. 2012 Oct;367(15):1397-406.

2 Malik VS, Pan A, Willett WC, Hu FB. Sugar-sweetened beverages and weight gain in children and adults: a systematic review and meta-analysis. Am J Clin Nutr. 2013 Oct;98(4):1084-102.

3 Ebbeling CB, Feldman HA, Osganian SK, Chomitz VR, Ellenbogen SJ, Ludwig DS. Effects of decreasing sugarsweetened beverage consumption on body weight in adolescents: a randomized, controlled pilot study. Pediatrics. 2006 Mar;117(3):673-80.

4 Ebbeling CB, Feldman HA, Chomitz VR, Antonelli TA, Gortmaker SL, Osganian SK, et al. A randomized trial of sugar-sweetened beverages and adolescent body weight. N Engl J Med. 2012 Oct;367(15):1407-16.

5 Imamura F, O'Connor L, Ye Z, Mursu J, Hayashino Y, Bhupathiraju SN, et al. Consumption of sugar sweetened beverages, artificially sweetened beverages, and fruit juice and incidence of type 2 diabetes: systematic review, meta-analysis, and estimation of population attributable fraction. BMJ. 2015 Jul;351:h3576.

6 Malik VS, Popkin BM, Bray GA, Després JP, Hu FB. Sugar-sweetened beverages, obesity, type 2 diabetes mellitus, and cardiovascular disease risk. Circulation. 2010 Mar;121(11):1356-64.

7 Vartanian LR, Schwartz MB, Brownell KD. Effects of soft drink consumption on nutrition and health: a systematic review and meta-analysis. Am J Public Health. 2007 Apr;97(4):667-75.

8 Singh GM, Micha R, Khatibzadeh S, Shi P, Lim S, Andrews KG, et al.; Global Burden of Diseases Nutrition and Chronic Diseases Expert Group (NutriCoDE). Global, regional, and national consumption of sugar-sweetened beverages, fruit juices, and milk: a systematic assessment of beverage intake in 187 countries. PLoS One. 2015 Aug; 10(8):e0124845.

9 Kit BK, Fakhouri TH, Park S, Nielsen SJ, Ogden CL. Trends in sugar-sweetened beverage consumption among youth and adults in the United States: 1999-2010. Am J Clin Nutr. 2013 Jul;98(1):180-8.

10 Gebremariam MK, Lien N, Nianogo RA, Arah OA. Mediators of socioeconomic differences in adiposity among youth: a systematic review. Obes Rev. 2017 Aug;18(8):880-98.

11 Hawkes C, Jewell J, Allen K. A food policy package for healthy diets and the prevention of obesity and dietrelated non-communicable diseases: the NOURISHING framework. Obes Rev. 2013 Nov;14 Suppl 2:159-68.

12 Abdel Rahman A, Jomaa L, Kahale LA, Adair P, Pine C. Effectiveness of behavioral interventions to reduce the intake of sugar-sweetened beverages in children and adolescents: a systematic review and meta-analysis. Nutr Rev. 2018 Feb; 76(2):88-107.

13 Vargas-Garcia EJ, Evans CE, Prestwich A, Sykes-Muskett BJ, Hooson J, Cade JE. Interventions to reduce consumption of sugar-sweetened beverages or increase water intake: evidence from a systematic review and meta-analysis. Obes Rev. 2017 Nov;18(11):1350-63.

14 Vercammen KA, Frelier JM, Lowery CM, McGlone ME, Ebbeling CB, Bleich SN. A systematic review of strategies to reduce sugar-sweetened beverage consumption among 0-year to 5-year olds. Obes Rev. 2018 Nov;19(11):1504-24.

15 Redondo M, Hernández-Aguado I, Lumbreras B. The impact of the tax on sweetened beverages: a systematic review. Am J Clin Nutr. 2018 Sep;108(3):548-63.

16 von Philipsborn P, Stratil JM, Burns J, Busert LK, Pfadenhauer LM, Polus S, et al. Environmental interventions to reduce the consumption of sugar-sweetened beverages and their effects on health. Cochrane Database Syst Rev. 2019 Jun;6:CD012292.

17 von Philipsborn P, Stratil J, Burns J, Busert LK, Pfadenhauer LM, Polus S, et al. Environmental interventions to reduce the consumption of sugar-sweetened beverages and their effects on health. Cochrane Database Syst Rev. 2019 Jun;6(6):CD012292.

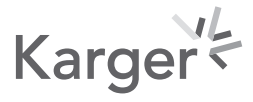




\begin{tabular}{|c|c|}
\hline \multicolumn{2}{|l|}{ Obes Facts 2020;13:397-417 } \\
\hline DOI: $10.1159 / 000508843$ & $\begin{array}{l}\text { (c) } 2020 \text { The Author(s). Published by S. Karger AG, Basel } \\
\text { www.karger.com/ofa }\end{array}$ \\
\hline
\end{tabular}

18 Cochrane Effective Practice and Organisation of Care (EPOC): EPOC resources for review authors [cited Oct 1, 2019]. Available from: https://epoc.cochrane.org/resources/epoc-resources-review-authors.

19 Heise TL, Katikireddi SV, Pega F, Gartlehner G, Fenton C, Griebler U, et al. Taxation of sugar-sweetened beverages for reducing their consumption and preventing obesity or other adverse health outcomes. Cochrane Database Syst Rev. 2016. https://doi.org/10.1002/14651858.CD012319.

20 USDA. US Department of Agriculture. 2015 - 2020 Dietary Guidelines for Americans, U.S. Department of Health and Human Services and U.S. Department of Agriculture [cited Oct 1, 2019]. Available from: http:// health.gov/dietaryguidelines/2015/.

21 Council on School HealthCommittee on Nutrition. Snacks, sweetened beverages, added sugars, and schools. Pediatrics. 2015 Mar;135(3):575-83.

22 Auerbach BJ, Dibey S, Vallila-Buchman P, Kratz M, Krieger J. Review of 100\% Fruit Juice and Chronic Health Conditions: Implications for Sugar-Sweetened Beverage Policy. Adv Nutr. 2018 Mar;9(2):78-85.

23 Hultcrantz M, Rind D, Akl EA, Treweek S, Mustafa RA, Iorio A, et al. The GRADE Working Group clarifies the construct of certainty of evidence. J Clin Epidemiol. 2017 Jul;87:4-13.

24 Visscher TL, van Hal WC, Blokdijk L, Seidell JC, Renders CM, Bemelmans WJ. Feasibility and impact of placing water coolers on sales of sugar-sweetened beverages in Dutch secondary school canteens. Obes Facts. 2010;3(2):109-15.

25 Bollinger B, Leslie P, Sorensen A. Calorie posting in chain restaurants. Am Econ J Econ Policy. 2011;3(1): 91-128.

26 Boelsen-Robinson T, Backholer K, Corben K, Blake MR, Palermo C, Peeters A. The effect of a change to healthy vending in a major Australian health service on sales of healthy and unhealthy food and beverages. Appetite. 2017 Jul;114:73-81.

27 Hartigan P, Patton-Ku D, Fidler C, Boutelle KN. Rethink your drink: reducing sugar-sweetened beverage sales in a children's hospital. Health Promot Pract. 2017 Mar;18(2):238-44.

28 Cornelsen L, Mytton OT, Adams J, Gasparrini A, Iskander D, Knai C, et al. Change in non-alcoholic beverage sales following a 10-pence levy on sugar-sweetened beverages within a national chain of restaurants in the UK: interrupted time series analysis of a natural experiment. J Epidemiol Community Health. 2017 Nov; 71(11):1107-12.

29 Blake MR, Peeters A, Lancsar E, Boelsen-Robinson T, Corben K, Stevenson CE, et al. Retailer-led sugarsweetened beverage price increase reduces purchases in a hospital convenience store in Melbourne, Australia: a mixed methods evaluation. J Acad Nutr Diet. 2018 Jun;118(6):1027-36.e8.

30 Breeze P, Womack R, Pryce R, Brennan A, Goyder E. The impact of a local sugar sweetened beverage health promotion and price increase on sales in public leisure centre facilities. PLoS One. 2018 May; 13(5):e0194637.

31 Foster GD, Karpyn A, Wojtanowski AC, Davis E, Weiss S, Brensinger C, et al. Placement and promotion strategies to increase sales of healthier products in supermarkets in low-income, ethnically diverse neighborhoods: a randomized controlled trial. Am J Clin Nutr. 2014 Jun;99(6):1359-68.

32 Collins A, Briefel R, Klerman J, Wolf A, Rowe G, Logan C, et al. Summer Electronic Benefit Transfer for Children (SEBTC) demonstration: summary report [cited Oct 1, 2019]. Available from: www.fns.usda.gov/ summer-electronic-benefit-transfer-children-sebtc-demonstration-summary-report.

33 Harnack L, Oakes JM, Elbel B, Beatty T, Rydell S, French S. Effects of subsidies and prohibitions on nutrition in a food benefit program: a randomized clinical trial. JAMA Intern Med. 2016 Nov;176(11):1610-8.

34 Olsho LE, Klerman JA, Wilde PE, Bartlett S. Financial incentives increase fruit and vegetable intake among Supplemental Nutrition Assistance Program participants: a randomized controlled trial of the USDA Healthy Incentives Pilot. Am J Clin Nutr. 2016 Aug;104(2):423-35.

35 Schwartz MB, Schneider GE, Choi YY, Li X, Harris J, Andreyeva T, et al. Association of a Community Campaign for Better Beverage Choices With Beverage Purchases From Supermarkets. JAMA Intern Med. 2017 May; 177(5):666-74.

36 Albala C, Ebbeling CB, Cifuentes M, Lera L, Bustos N, Ludwig DS. Effects of replacing the habitual consumption of sugar-sweetened beverages with milk in Chilean children. Am J Clin Nutr. 2008 Sep;88(3):605-11.

37 Anand SS, Davis AD, Ahmed R, Jacobs R, Xie C, Hill A, et al.; SHARE-AP ACTION Investigators. A family-based intervention to promote healthy lifestyles in an aboriginal community in Canada. Can J Public Health. 2007 Nov-Dec;98(6):447-52.

38 Hernández-Cordero S, Barquera S, Rodríguez-Ramírez S, Villanueva-Borbolla MA, González de Cossio T, Dommarco JR, et al. Substituting water for sugar-sweetened beverages reduces circulating triglycerides and the prevalence of metabolic syndrome in obese but not in overweight Mexican women in a randomized controlled trial. J Nutr. 2014 Nov;144(11):1742-52.

39 Tate DF, Turner-McGrievy G, Lyons E, Stevens J, Erickson K, Polzien K, et al. Replacing caloric beverages with water or diet beverages for weight loss in adults: main results of the Choose Healthy Options Consciously Everyday (CHOICE) randomized clinical trial. Am J Clin Nutr. 2012 Mar;95(3):555-63.

40 Cawley J, Sweeney MJ, Sobal J, Just DR, Kaiser HM, Schulze WD, et al. The impact of a supermarket nutrition rating system on purchases of nutritious and less nutritious foods. Public Health Nutr. 2015 Jan;18(1): 8-14. 


\begin{tabular}{l|l}
\hline Obes Facts 2020;13:397-417 \\
\hline DOI: 10.1159/000508843 & $\begin{array}{l}\text { @ 2020 The Author(s). Published by S. Karger AG, Basel } \\
\text { www.karger.com/ofa }\end{array}$ \\
\hline
\end{tabular}

41 Hobin E, Bollinger B, Sacco J, Liebman E, Vanderlee L, Zuo F, et al. Consumers' response to an on-shelf nutrition labelling system in supermarkets: evidence to inform policy and practice. Milbank Q. 2017 Sep; 95(3):494-534.

42 Cradock AL, McHugh A, Mont-Ferguson H, Grant L, Barrett JL, Wang YC, et al. Effect of school district policy change on consumption of sugar-sweetened beverages among high school students, Boston, Massachusetts, 2004-2006. Prev Chronic Dis. 2011 Jul;8(4):A74.

43 Blum JE, Davee AM, Beaudoin CM, Jenkins PL, Kaley LA, Wigand DA. Reduced availability of sugar-sweetened beverages and diet soda has a limited impact on beverage consumption patterns in Maine high school youth. J Nutr Educ Behav. 2008 Nov-Dec;40(6):341-7.

44 Bauhoff $S$. The effect of school district nutrition policies on dietary intake and overweight: a synthetic control approach. Econ Hum Biol. 2014 Jan;12:45-55.

45 Schwartz MB, Novak SA, Fiore SS. The impact of removing snacks of low nutritional value from middle schools. Health Educ Behav. 2009 Dec;36(6):999-1011.

46 Peters J, Beck J, Lande J, Pan Z, Cardel M, Ayoob K, et al. Using healthy defaults in Walt Disney World restaurants to improve nutritional choices. J Assoc Consum Res. 2016 Jan;1(1):92-103.

47 Elbel B, Mijanovich T, Abrams C, Cantor J, Dunn L, Nonas C, et al. A water availability intervention in New York City public schools: influence on youths' water and milk behaviors. Am J Public Health. 2015 Feb; 105(2):365-72.

48 Muckelbauer R, Libuda L, Clausen K, Toschke AM, Reinehr T, Kersting M. Promotion and provision of drinking water in schools for overweight prevention: randomized, controlled cluster trial. Pediatrics. 2009 Apr;123(4):e661-7.

49 van de Gaar VM, Jansen W, van Grieken A, Borsboom G, Kremers S, Raat H. Effects of an intervention aimed at reducing the intake of sugar-sweetened beverages in primary school children: a controlled trial. Int J Behav Nutr Phys Act. 2014 Jul;11(1):98.

50 Øverby NC, Klepp KI, Bere E. Introduction of a school fruit program is associated with reduced frequency of consumption of unhealthy snacks. Am J Clin Nutr. 2012;96:1100-03.

51 Sturm R, Hattori A. Diet and obesity in Los Angeles County 2007-2012: is there a measurable effect of the 2008 "Fast-Food Ban"? Soc Sci Med. 2015 May;133:205-11.

52 Minaker LM, Olstad DL, MacKenzie G, Nguyen N, Azagba S, Cook BE, et al. An evaluation of the impact of a restrictive retail food environment intervention in a rural community pharmacy setting. BMC Public Health. 2016 Jul;16(1):586.

53 Elbel B, Mijanovich T, Dixon LB, Abrams C, Weitzman B, Kersh R, et al. Calorie labeling, fast food purchasing and restaurant visits. Obesity (Silver Spring). 2013 Nov;21(11):2172-9.

54 Finkelstein EA, Strombotne KL, Chan NL, Krieger J. Mandatory menu labeling in one fast-food chain in King County, Washington. Am J Prev Med. 2011 Feb;40(2):122-7.

55 Ball K, McNaughton SA, Le HN, Gold L, Ni Mhurchu C, Abbott G, et al. Influence of price discounts and skillbuilding strategies on purchase and consumption of healthy food and beverages: outcomes of the Supermarket Healthy Eating for Life randomized controlled trial. Am J Clin Nutr. 2015 May;101(5):1055-64.

56 Ni Mhurchu C, Blakely T, Jiang Y, Eyles HC, Rodgers A. Effects of price discounts and tailored nutrition education on supermarket purchases: a randomized controlled trial. Am J Clin Nutr. 2010 Mar;91(3):73647.

57 Franckle RL, Levy DE, Macias-Navarro L, Rimm EB, Thorndike AN. Traffic-light labels and financial incentives to reduce sugar-sweetened beverage purchases by low-income Latino families: a randomized controlled trial. Public Health Nutr. 2018 Jun;21(8):1426-34.

58 Ng SW, Popkin BM. The Healthy Wight Commitment Foundation pledge: calories purchased by U.S. households with children, 2000-2012. Am J Prev Med. 2014b 0ct;47(4):520-30.

59 Ng SW, Slining MM, Popkin BM. The Healthy Weight Commitment Foundation pledge: calories sold from U.S. consumer packaged goods, 2007-2012. Am J Prev Med. 2014a Oct;47(4):508-19.

60 Taillie LS, Ng SW, Popkin BM. Gains made by Walmart's Healthier Food Initiative mirror preexisting trends. Health Aff (Millwood). 2015 Nov;34(11):1869-76.

61 Ermetici F, Zelaschi RF, Briganti S, Dozio E, Gaeta M, Ambrogi F, et al. Association between a school-based intervention and adiposity outcomes in adolescents: the Italian "EAT" project. Obesity (Silver Spring). 2016 Mar;24(3):687-95.

62 French SA, Hannan PJ, Harnack LJ, Mitchell NR, Toomey TL, Gerlach A. Pricing and availability intervention in vending machines at four bus garages. J Occup Environ Med. 2010 Jan;52(Suppl 1):S29-33.

63 Baker P, Friel S, Schram A, Labonte R. Trade and investment liberalization, food systems change and highly processed food consumption: A natural experiment contrasting the soft-drink markets of Peru and Bolivia. Global Health. 2016 Jun;12(1):24.

64 Schram A, Labonte R, Baker P, Friel S, Reeves A, Stuckler D. The role of trade and investment liberalization in the sugar-sweetened carbonated beverages market: a natural experiment contrasting Vietnam and the Philippines. Global Health. 2015 Oct;11(1):41.

65 Waehrer G, Deb P, Decker SL. Did the 2009 American Recovery and Reinvestment Act affect dietary intake of low-income individuals? Econ Hum Biol. 2015 Dec;19:170-83.

66 Schwartz AE, Leardo M, Aneja S, Elbel B. Effect of a school-based water intervention on child body mass index and obesity. JAMA Pediatr. 2016 Mar;170(3):220-6.

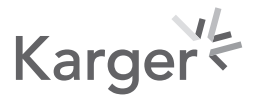


67 Hua SV, Kimmel L, Van Emmenes M, Taherian R, Remer G, Millman A, et al. Health promotion and healthier products increase vending purchases: a randomized factorial trial. J Acad Nutr Diet. 2017 Jul;117(7): 1057-65.

68 Brimblecombe J, Ferguson M, Chatfield MD, Liberato SC, Gunther A, Ball K, et al.; SHOP@RIC research collaborative. Effect of a price discount and consumer education strategy on food and beverage purchases in remote Indigenous Australia: a stepped-wedge randomised controlled trial. Lancet Public Health. 2017 Feb;2(2):e82-95.

69 Huang R, Kiesel K. Does limited access at school result in compensation at home? the effect of soft drink bans in schools on purchase patterns outside of schools. Eur Rev Agric Econ. 2012;39(5):797-820.

70 Lichtman-Sadot S. Does banning carbonated beverages in schools decrease student consumption? J Public Econ. 2016;140:30-50.

71 Da Costa FF, De Assis MA, Gonzalez-Chica D, Bernardo C, De Barros MV, Nahas MV, et al. Effect of schoolbased intervention on diet in high school students. Rev Bras Cineantropom Desempenho Hum. 2014;16: 36-45.

72 Simons M, Brug J, Chinapaw MJ, de Boer M, Seidell J, de Vet E. Replacing non-active video gaming by active video gaming to prevent excessive weight gain in adolescents. PLoS One. 2015 Jul;10(7):e0126023.

73 Siegel RM, Hudgens M, Annekin A, Simmons K, Shelly J, Bell I, et al. A two-tiered school cafeteria intervention of emoticons and small prizes increased healthful food selection by over $300 \%$. Int J Food Nutr Sci. 2016;5(3):60-66.

74 Siegel RM, Geoppinger R, Emerson MM, Barnes A, Shelly J, Crumpton M, et al. Small prizes to improve food selection in inner city elementary schools. J Pediatr Child Nutr. 2016;3:100117.

75 Cohen JF, Richardson SA, Cluggish SA, Parker E, Catalano PJ, Rimm EB. Effects of choice architecture and chef-enhanced meals on the selection and consumption of healthier school foods: a randomized clinical trial. JAMA Pediatr. 2015 May; 169(5):431-7.

76 Emerson M, Hudgens M, Barnes A, Hiller E, Robison D, Kipp R, et al. Small prizes increased plain milk and vegetable selection by elementary school children without adversely affecting total milk purchase. Beverages. 2017;3(4):14.

77 Hendy HM, Williams KE, Camise TS. Kid's Choice Program improves weight management behaviors and weight status in school children. Appetite. 2011 Apr;56(2):484-94.

78 Castillo Martinez L. Sugar-sweetened beverage intake substitution by water to prevent overweight in Mexican children [cited Apr 1, 2019]. Available from: https://clinicaltrials.gov/ct2/show/NCT03069274.

79 Swanson M. Appalachians together restoring the eating environment: improving healthy diet in rural Appalachian Kentucky [cited Apr 1, 2019]. Available from: https://clinicaltrials.gov/ct2/show/ NCT02996422.

80 Patel AI. School water access, food and beverage intake, and obesity [cited Apr 1, 2019]. Available from: https://clinicaltrials.gov/ct2/show/NCT03181971.

81 Kajons N. Thirsty? Choose water! Behavioural interventions and water stations in secondary schools [cited Apr 1, 2019]. Available from: www.anzctr.org.au/Trial/Registration/TrialReview.aspx?id=374487.

82 Goran MI. Improving the eating habits of mother and her infant via sugar reduction (MAMITA) [cited Apr 1, 2019]. Available from: https://clinicaltrials.gov/ct2/show/NCT03141346.

83 Reeves G. Improving cardiometabolic health of youth on antipsychotic medication [cited Apr 1, 2019]. Available from: https://clinicaltrials.gov/ct2/show/NCT02877823.

84 Wolfenden L. A cluster randomised controlled trial of a sugar-sweetened beverage intervention in secondary schools [cited Apr 1, 2019]. Available from: www.anzctr.org.au/Trial/Registration/TrialReview.aspx?id=373480.

85 Schmidt J. The impact of workplace food and beverage choices on health and wellness [cited Apr 1, 2019]. Available from: https://clinicaltrials.gov/ct2/show/NCT03431051.

86 John L. Do taxes reduce the purchasing of soda? [cited Apr 1, 2019]. Available from: https://clinicaltrials. gov/ct2/show/NCT02914821.

87 University Hospital Southampton NHS Foundation Trust. Women's responses to adjusted product placement and its effects on diet - 1 (WRAPPED1) [cited Apr 1, 2019]. Available from: https://clinicaltrials. gov/ct2/show/NCT03518151.

88 Rutter H, Savona N, Glonti K, Bibby J, Cummins S, Finegood DT, et al. The need for a complex systems model of evidence for public health. Lancet. 2017 Dec;390(10112):2602-4.

89 Avery A, Bostock L, McCullough F. A systematic review investigating interventions that can help reduce consumption of sugar-sweetened beverages in children leading to changes in body fatness. J Hum Nutr Diet. 2015 Jan;28 Suppl 1:52-64.

90 Lane H, Porter K, Estabrooks P, Zoellner J. A Systematic Review to Assess Sugar-Sweetened Beverage Interventions for Children and Adolescents across the Socioecological Model. J Acad Nutr Diet. 2016 Aug; 116(8):1295-1307.e6.

91 Vézina-Im LA, Beaulieu D, Bélanger-Gravel A, Boucher D, Sirois C, Dugas M, et al. Efficacy of school-based interventions aimed at decreasing sugar-sweetened beverage consumption among adolescents: a systematic review. Public Health Nutr. 2017 Sep;20(13):2416-31. 
92 Mazarello Paes V, Hesketh K, O’Malley C, Moore H, Summerbell C, Griffin S, et al. Determinants of sugarsweetened beverage consumption in young children: a systematic review. Obes Rev. 2015 Nov;16(11): 903-13.

93 Hennessy M, Bleakley A, Piotrowski JT, Mallya G, Jordan A. Sugar-sweetened beverage consumption by adult caregivers and their children: the role of drink features and advertising exposure. Health Educ Behav. 2015 Oct;42(5):677-86.

94 Mantzari E, Hollands GJ, Pechey R, Jebb S, Marteau TM. Impact of bottle size on in-home consumption of sugar-sweetened beverages: a feasibility and acceptability study. BMC Public Health. 2017 Apr; 17(1):304.

95 VanEpps EM, Roberto CA. The influence of sugar-sweetened beverage warnings: a randomized trial of adolescents' choices and beliefs. Am J Prev Med. 2016 Nov;51(5):664-72.

96 Jones A, Magnusson R, Swinburn B, Webster J, Wood A, Sacks G, et al. Designing a healthy food partnership: lessons from the Australian Food and Health Dialogue. BMC Public Health. 2016 Jul;16(1):651.

97 Glickman D, Veneman AM. The essential role of food and farm policy in improving health. Health Aff (Millwood). 2013 Sep;32(9):1519-21.

98 Ortega AN, Albert SL, Chan-Golston AM, Langellier BA, Glik DC, Belin TR, et al. Substantial improvements not seen in health behaviors following corner store conversions in two Latino food swamps. BMC Public Health. 2016 May;16(1):389. 